\title{
Female and younger subjects have lower adherence in PrEP trials: a meta-analysis with implications for the uptake of PrEP service to prevent HIV
}

\author{
Ke Yun, ${ }^{1,2}$ Jun-jie $\mathrm{Xu}_{1}{ }^{1,2}$ Jing Zhang, ${ }_{1,2}$ Jia-ming $\mathrm{Li}^{1,2}{ }^{1,2}$ Qing-hai $\mathrm{Hu}_{1}{ }^{1,2}$ Zhen-xing $\mathrm{Chu}_{1}{ }^{1,2}$ \\ Yong-jun Jiang, ${ }^{1,2}$ Wen-qing Geng, ${ }^{1,2}$ Hong Shang, ${ }^{1,2}$ Ning Wang ${ }^{1,3}$
}

\begin{abstract}
- Additional material is published online only. To view please visit the journal online (http://dx.doi.org/10.1136/ sextrans-2017-053217)

${ }^{1}$ Key Laboratory of AIDS Immunology of National Health and Family Planning Commission, Department of Laboratory Medicine, The First Affiliated Hospital, China Medical University, Shenyang, China

${ }^{2}$ Collaborative Innovation Center for Diagnosis and Treatment of Infectious Diseases, Hangzhou, China

${ }^{3}$ National Center for AIDS/STI Control and Prevention, Chinese Center for Disease Control and Prevention, Beijing, China
\end{abstract}

\section{Correspondence to}

Hong Shang, Key Laboratory of AIDS Immunology of National Health and Family Planning Commission, Department of Laboratory Medicine, The First Affiliated Hospital, China Medical University, No 155, Nanjingbei Street, Heping District, Shenyang, Liaoning Province 110001, China: hongshang100@hotmail. com and Ning Wang, National Center for AIDS/STI Control and Prevention, Chinese Center for Disease Control and Prevention, No. 155 Changbai Road Changping District, Beijing 102206, China; wangnbj@ 163.com

$\mathrm{KY}$ and $\mathrm{J}-\mathrm{X}$ contributed equally.

Received 1 April 2017

Revised 8 June 2017

Accepted 2 July 2017

Published Online First 29 July 2017

Check for updates

To cite: Yun $\mathrm{K}, \mathrm{Xu} \mathrm{J}$, Zhang J, et al. Sex Transm Infect

2018:94:163-168.

\begin{abstract}
Objective To estimate the medicine-taking compliance (MTC) level, explore its facilitators and barriers, and quantify the association between MTC level and pre-exposure prophylaxis (PrEP) protective efficacy in individuals at risk of acquiring HIV being administered oral PrEP.
\end{abstract}

Design Meta-analysis.

Data sources We searched PubMed, Cochrane and Embase databases for published randomized controlled trials (RCTs) pertaining to MTC of oral PrEP for HIV prevention up to 16 January 2017.

Review methods The pooled proportion of MTC and risk ratio (RR) of HIV incidences between intervention group and control group were estimated.

Results We identified 10 eligible studies with 24193 participants. The overall pooled MTC for oral HIV PrEP was $59.9 \%$ (95\% Cl $43.1 \%$ to $74.6 \%$ ). Subgroup analyses revealed that the MTC level of participants aged $<30$ years was lower than those equal or older than 30 years (34.9\% vs $69.6 \%, p<0.001)$; those studies that enrolled only women as participants had lower MTC than those only recruiting either only men or both men and women (31.3\% vs $71.7 \%$ and $31.3 \%$ vs $71.0 \%$, all $\mathrm{p}<0.01)$. Additionally, the HIV infection risk increased as the MTC level declines, with the incidence RRs being 0.28 ( $95 \% \mathrm{Cl} 0.19$ to 0.41$), 0.42$ ( $95 \% \mathrm{Cl} 0.29$ to 0.62$)$ and $0.75(95 \% \mathrm{Cl} 0.45$ to 1.25$)$ in the good ( $\geq 80 \%)$, moderate $(60 \% \sim 80 \%)$ and poor $(<60 \%)$ MTC subgroups, respectively (linear trend test $p<0.01$ ). Conclusion According to the pooled proportion, the MTC of oral HIV PrEP is almost moderate, and its proportion in women and younger participants was relatively low. The protective efficacy of oral PrEP for HIV prevention increased with MTC level. These findings indicated that it is necessary to identify measures to enhance MTC of oral PrEP in future clinical usage, especially in women and younger participants with high HIV infection risk.

\section{INTRODUCTION}

Preventing the spread of HIV remains a worldwide major public health challenge. ${ }^{1}$ As the safety and efficacy of antiretroviral drugs have increased, antiretroviral pre-exposure prophylaxis (PrEP) for individuals at high risk of HIV infection is considered a hopeful new HIV prevention strategy. In recent years, the Centers for Disease Control and Prevention (CDC) in the USA has recommended daily oral PrEP to reduce the HIV infection risk of high-risk populations, ${ }^{2}$ such as men who have sex with men (MSM), certain heterosexual groups and people who inject drugs (PWID). However, the efficacy of daily oral PrEP at reducing the risk of HIV infection has varied in these studies. For example, a clinical trial performed in African women using Truvada (a combination of emtricitabine (FTC) $200 \mathrm{mg}$ and tenofovir disoproxil fumarate (TDF) $300 \mathrm{mg}$ ) did not reveal any significant efficacy of HIV prevention; it was speculated that inconsistent medicine-taking compliance (MTC) might be the reason for the failure. ${ }^{34}$ Good MTC is crucial for the success of PrEP in HIV prevention; in fact it has been claimed to be the main determinant of PrEP efficacy. ${ }^{5}$ Nonetheless, it remains unclear which factors influence MTC in individuals receiving PrEP for HIV prevention. Additionally, the quantified relationship betweenPrEP MTC and its protective efficacy has not been systematically evaluated.

Self-reported MTC might not be reliable and may suffer from the so-called social desirability bias. ${ }^{6}$ Instead, drug concentrations measured either from serum $^{4}$ and dried blood spots ${ }^{7}$ have emerged as an objective way of measuring MTC in PrEP studies. Thus, the purpose of our meta-analysis was threefold: (1) to determine the level of MTC in PrEP based on assayed blood drug levels, (2) to explore facilitators and barriers to MTC and (3) to clarify the association between PrEP efficacy and MTC.

\section{METHODS}

\section{Study selection}

This meta-analysis was conducted according to the Preferred Reporting Items for Systematic Reviews and Meta-Analyses guidelines. ${ }^{8}$ The PubMed, Cochrane and Embase databases were searched through 16 January 2017 to identify relevant articles. The free text and medical subject headings $(\mathrm{MeSH})$ terms pertaining to MTC of oral PrEP were used to search potentially relevant literatures. Studies that met the following criteria were included in our meta-analysis: (1) randomised controlled trial (RCT) of oral PrEP efficacy study on HIV; and (2) reported quantitative data of oral PrEP MTC containing TDF/FTC to prevent HIV infection. MTC levels were defined as the percentage of HIV-negative participants receiving PrEP with detectable levels of study medication in their blood sampled. Duplicate reports, qualitative studies and studies that did not report plasma drug 
concentration measurement were excluded from this meta-analysis. All included studies were written in English or Chinese and contained the relevant data for the integration of oral PrEP MTC proportion and PrEP protective efficacy.

\section{Data extraction}

All studies describing MTC of oral PrEP were targeted. Citation titles and/or abstracts were screened, and relevant original studies were read in full. Data of included studies were extracted by two reviewers independently (KY and JML). The following information was extracted: first author, publication date, study location, sample size, number of individuals in whom data about MTC were available, sample size and incidence data of HIV infection. Data extraction disagreements between the two reviewers were reconciled by group discussion.

\section{Quality assessment}

The methodological quality of the individual randomised clinical trial was assessed using the Jadad scale, ${ }^{9}$ consisting of three parameters of quality including randomisation, blinding and withdrawals. Studies scoring 1 or 2 points are considered low quality, whereas those scoring 3-5 points are assessed as high quality.

\section{Statistical analysis}

If heterogeneity test was significant, random effect model was used, otherwise fixed effect model was used. ${ }^{10}$ Statistical heterogeneity was quantified with the $\mathrm{I}^{2}$ statistic (low $<25 \%$, moderate $=25 \%-50 \%$, high $>50 \%)$. $^{11}$ In order to clarify the source of heterogeneity, subgroup analysis and meta regression were conducted according to the following factors: age $(<30$ years vs $\geq 30$ years), study drug (TDF vs TDF/FTC), transmission mode (needles/syringe, rectal, vaginal and vaginal/penile), sample size ( $<1500$ vs $\geq 1500$ ), follow-up months ( $\leq 24$ vs $\leq 36$ vs $>36$ ), medication intensity (daily vs intermittent), biological sex (women, men or both genders), clinical trial type (non-openlabel RCT vs open-label RCT) and Jadad scale score ( $<5$ points vs 5 points). To confirm that our findings were not biased by any single study, we compared the overall pooled proportion with estimates obtained after iterations by applying a leave-one-out sensitivity analysis. In addition, potential publication bias was examined using Egger's and Begger's tests. When we integrated the protective efficacy in the different MTC subgroups, we divided MTC levels into three groups reflecting good $(\geq 80 \%)$, moderate $(60 \% \sim 80 \%)$ or poor $(<60 \%)$ MTC according to the break point of the relationship curve using local weighted regression (loess) method.

\section{RESULTS}

\section{Description of eligible studies}

A total of 1594 articles were selected and screened according to the inclusion and exclusion criteria (online supplementary figure 1). Descriptive summary of the final 10 studies with 13 independent PrEP efficacy estimates, which used either TDF or the combination of TDF-FTC as the intervention drug for HIV prevention, is shown in table 1 . The sample size in these studies ranged from 400 to 4969 , publication year was from 2007 to 2016, and the follow-up months ranged from 12 to 84; the study populations included IDU, serodiscordant couples, MSM and transgender women, high-risk women and/or heterosexual men living in a high HIV epidemic region; PrEP medication intensity included either a daily regimen or an intermittent regimen.

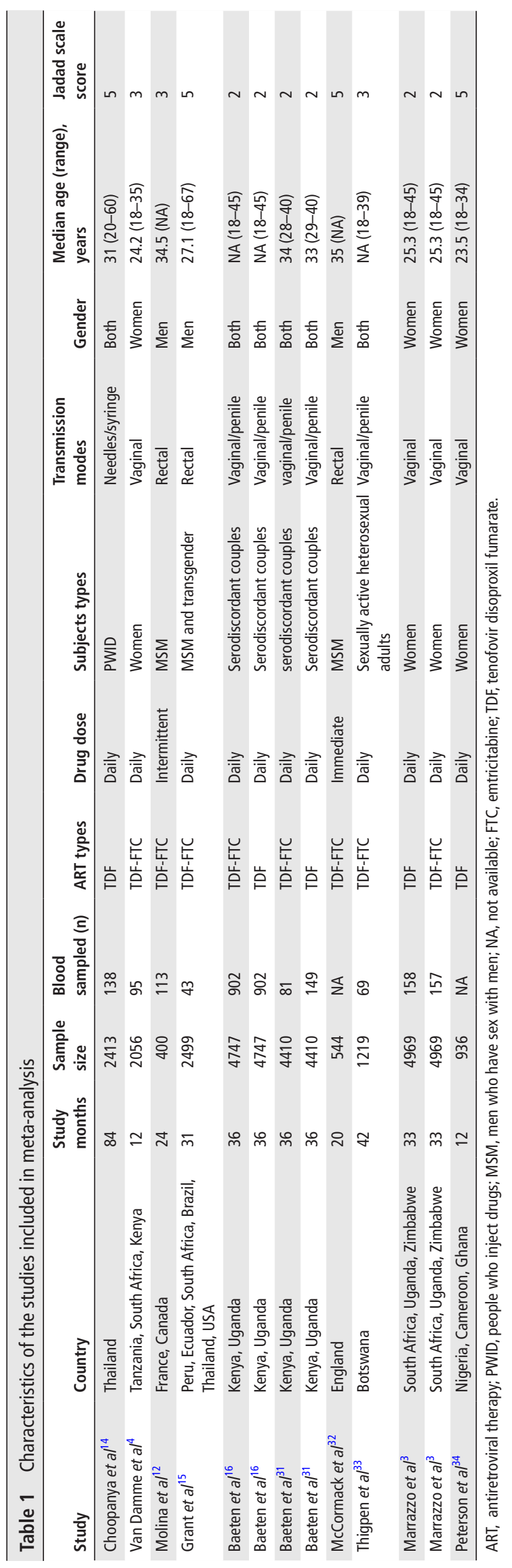


Table 2 Subgroup analysis of factors associated with MTC proportion in PrEP trials

\begin{tabular}{|c|c|c|c|c|c|c|c|}
\hline Variable & Subgroup & Study (n) & MTC proportion $(95 \% \mathrm{Cl})$ & $I^{2}(\%)$ & $\begin{array}{l}\text { Meta-regression } \\
\text { coefficient }\end{array}$ & SE & $\mathrm{p}$ Value \\
\hline \multirow[t]{2}{*}{ Age (years) } & $<30$ & 4 & $34.9 \%(27.6 \%$ to $43.1 \%)$ & 64.50 & Ref & & \\
\hline & $\geq 30$ & 4 & $69.6 \%(56.1 \%$ to $80.4 \%)$ & 87.70 & 1.42 & 0.32 & $<0.001$ \\
\hline \multirow[t]{3}{*}{ Gender } & Women & 3 & $31.3 \%(27.0 \%$ to $36.0 \%)$ & 0 & Ref & & \\
\hline & Men & 2 & $71.7 \%(31.2 \%$ to $93.4 \%)$ & 94.60 & 1.71 & 0.57 & 0.0023 \\
\hline & Both & 5 & $71.0 \%(58.7 \%$ to $80.9 \%)$ & 92.30 & 1.66 & 0.43 & 0.0001 \\
\hline \multirow[t]{5}{*}{ Population } & High-risk women & 3 & $31.3 \%(27.0 \%$ to $36.0 \%)$ & 0 & Ref & & \\
\hline & PWID & 1 & $67.4 \%(59.2 \%$ to $74.7 \%)$ & - & 1.49 & 0.76 & 0.0494 \\
\hline & Sexually active heterosexual adults & 1 & $79.7 \%(68.6 \%$ to $87.6 \%)$ & - & 2.13 & 0.79 & 0.0073 \\
\hline & MSM and transgender & 2 & $71.7 \%(31.2 \%$ to $93.4 \%)$ & 94.60 & 1.71 & 0.62 & 0.0059 \\
\hline & Serodiscordant couples & 3 & $69.2 \%$ (49.2\% to $83.8 \%)$ & 95.50 & 1.58 & 0.53 & 0.0032 \\
\hline \multirow[t]{4}{*}{ Transmission mode } & Vaginal & 3 & $31.3 \%(27.0 \%$ to $36.0 \%)$ & 0 & Ref & & \\
\hline & Needles/syringe & 1 & $67.4 \%(59.2 \%$ to $74.7 \%)$ & - & 1.49 & 0.72 & 0.0382 \\
\hline & Vaginal/penile & 4 & $71.9 \%(56.6 \%$ to $83.3 \%)$ & 93.30 & 1.70 & 1.70 & 0.0004 \\
\hline & Rectal & 2 & $71.7 \%(31.2 \%$ to $93.4 \%)$ & 94.60 & 1.71 & 0.59 & 0.0038 \\
\hline \multirow[t]{2}{*}{ Medication intensity } & Daily & 9 & $56.1 \%(38.6 \%$ to $72.3 \%)$ & 97.30 & Ref & & \\
\hline & Intermittent & 1 & $85.8 \%(78.1 \%$ to $91.1 \%)$ & & 1.56 & 1.16 & 0.1806 \\
\hline \multirow[t]{2}{*}{ Drug type } & TDF & 3 & $55.3 \%(30.0 \%$ to $78.2 \%)$ & 96.40 & Ref & & \\
\hline & FTC/TDF & 7 & $61.8 \%(39.7 \%$ to $79.9 \%)$ & 97.40 & 0.27 & 0.79 & 0.7333 \\
\hline \multirow[t]{2}{*}{ Sample size } & $<1500$ & 2 & $83.3 \%(76.5 \%$ to $88.4 \%)$ & 13.70 & Ref & & \\
\hline & $\geq 1500$ & 8 & $52.8 \%(34.2 \%$ to $70.7 \%)$ & 97.60 & -1.47 & 0.87 & 0.0887 \\
\hline \multirow[t]{3}{*}{ Follow-up months } & $\leq 24$ & 2 & $65.2 \%(15.9 \%$ to $94.9 \%)$ & 97.80 & Ref & & \\
\hline & $\leq 36$ & 6 & $52.9 \%(29.8 \%$ to $74.8 \%)$ & 98.10 & -0.50 & 1.00 & 0.6128 \\
\hline & $>36$ & 2 & $73.2 \%(59.4 \%$ to $83.6 \%)$ & 70.30 & 0.42 & 1.22 & 0.7310 \\
\hline \multirow[t]{2}{*}{ Clinical trial type } & Non-open-label RCT & 7 & $64.0 \%(49.0 \%$ to $76.6 \%)$ & 95 & Ref & & \\
\hline & Open-label RCT & 3 & $50.1 \%(19.2 \%$ to $81.0 \%)$ & 97.50 & -0.58 & 0.65 & 0.3730 \\
\hline \multirow[t]{2}{*}{ Jadad scale score } & $<5$ & 8 & $59.9 \%(39.6 \%$ to $77.3 \%)$ & 97.80 & Ref & & \\
\hline & 5 & 2 & $60.6 \%(44.3 \%$ to $74.9 \%)$ & 72.70 & -0.01 & 0.91 & 0.9936 \\
\hline
\end{tabular}

PWID, people who inject drugs; MSM, men who have sex with men; MTC, medicine-taking compliance; PrEP, pre-exposure prophylaxis; RCT, randomised controlled trial; Ref, reference category; FTC, emtricitabine; TDF, tenofovir disoproxil fumarate.

\section{Pooled proportion of oral PrEP MTC and subgroup analysis}

There was substantial heterogeneity between studies $\left(\mathrm{I}^{2}=97.2 \%\right.$ $(\mathrm{Q}=318.35, \mathrm{p}<0.001))$ and therefore random effects model was used. The integrated findings from these included studies indicated that the estimated proportion of oral PrEP MTC was $59.9 \%$ (95\% CI $43.1 \%$ to $74.6 \%$ ) (online supplementary figure 2 ). The source of the heterogeneity was explored in subgroup analyses; the results revealed that the MTC of studies with participants aged $<30$ years was lower than the corresponding value in individuals older than 30 years $(34.9 \%$ (95\% CI $27.6 \%$ to $43.1 \%)$ vs $69.6 \%$ (95\% CI $56.1 \%$ to $80.4 \%)$, p $<0.001)$ ); studies including only women as participants had lower MTC than those that recruited either only men or both men and women as participants $(31.3 \%$ (95\% CI $27.0 \%$ to $36.0 \%)$ vs $71.7 \%$ (95\% CI $31.2 \%$ to $93.4 \%$ ) and $71.0 \%$ (95\% CI $58.7 \%$ to $80.9 \%$ ), all $\mathrm{p}<0.001)$. Identical results were also found from the perspective of high-risk populations and transmission routes. For example, the results showed that the MTC of sexually active heterosexual adults living in a high HIV prevalence country was the highest (79.7\% (95\% CI 68.6\% to $87.6 \%)$ ), followed by MSM/transgender (71.7\% (95\% CI 31.2\% to 93.4\%)), serodiscordant couples $(69.2 \%$ (95\% CI $49.2 \%$ to $83.8 \%)$ ) and IDU $(67.4 \%$ (95\% CI 59.2\%, 74.7\%)). MTC levels in studies where HIV would be transmitted through vaginal/penile, rectal and IDU were higher than those where transmission was via a vaginal transmission mode (71.9\% (95\% CI $56.6 \%$ to $83.3 \%$ ), $71.7 \%$ (95\% CI $31.2 \%$ to $93.4 \%$ ) and $67.4 \%$ (95\% CI $59.2 \%$ to $74.7 \%$ ) vs $31.3 \%$ (95\% CI $27.0 \%$ to $36.0 \%)$ ) (table 2 ).

\section{Sensitivity analysis and publication bias}

In the sensitivity analysis, the MTC proportion varied from 56\% to $63 \%$, which were all within the CI of the pooled estimate, and thus indicated that no individual study exerted undue influence on the overall outcome. The assessment of funnel plot asymmetry revealed that no potential publication bias was found among the included studies (Egger's test: $\mathrm{t}=-1.4578, \mathrm{p}=0.183$; Begger's test: $\mathrm{z}=0.4472, \mathrm{p}=0.6547)$.

\section{Association between MTC and PrEP efficacy for preventing HIV infection}

A total of 41930 person years (PYs) were followed, with 726 HIV infections being identified from 10 studies. Of these, 299 HIV infections were found in the intervention group (incidence, 1.27 (95\% CI 0.68 to 2.36) per 100 PYs) and 427 in the control group (incidence, 3.08 (95\% CI 2.15 to 4.40$)$ per $100 \mathrm{PYs}$ ). The pooled incidence risk ratio (RR) of oral PrEP for HIV infection was 0.45 (95\% CI 0.31 to 0.67 ) (online supplementary figure 3 ). The integrated results found that there was a positive correlation between MTC and PrEP protective efficacy (figure 1). Subgroup analysis demonstrated the following pooled HIV incidences of good, moderate and poor MTC subgroups, that is, $0.60(95 \%$ CI 0.42 to 0.84$), 0.65$ (95\% CI 0.35 to 1.21 ) and 2.80 (95\% CI 1.31 to 5.90$)$ per $100 \mathrm{PYs}$ in the treatment group, and 2.83 (95\% CI 1.59 to 4.99 ), 1.61 (95\% CI 0.59 to 4.35 ) and 4.15 (95\% CI 3.39 to 5.07) in the control group. Furthermore, an increased MTC level was associated with a significant decline in 


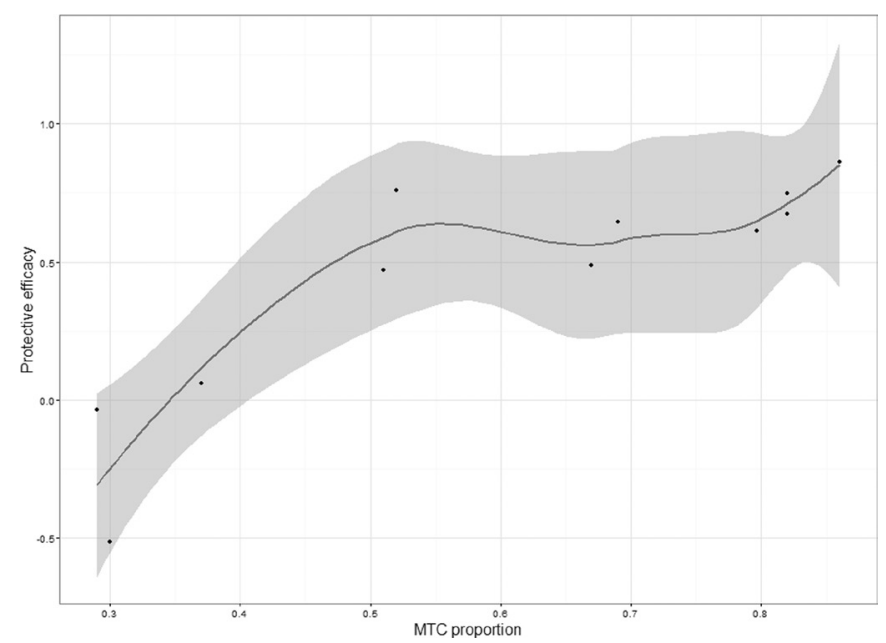

Figure 1 Summary revealing the non-linear relationship between pre-exposure prophylaxis medicine-taking compliance (MTC) and its protective efficacy.

the incidence RRs of HIV infection (poor MTC: 0.75 (95\% CI 0.45 to 1.25 ), moderate MTC: 0.42 (95\% CI 0.29 to 0.62 ) and good MTC: 0.28 (95\% CI 0.19 to 0.41$)$ ) (linear trend test $\mathrm{p}<0.01$ ) (table 3).

\section{DISCUSSION}

As far as we are aware, this is the first study to evaluate the integrated MTC proportion of oral PrEP for HIV prevention. We found that the pooled MTC proportion was 59.9\% (95\% CI $43.1 \%$ to $74.6 \%$ ) in individuals at high risk of acquiring HIV, which can be considered as moderate. The MTC level found here was lower than that revealed by self-administered questionnaires $(59.9 \%$ vs $72 \%),{ }^{12}$ highlighting the risk that results obtained by self-reporting may suffer from asocial desirability bias. $^{6}$ At present, animal experiments ${ }^{13}$ and several population clinical trials ${ }^{14-16}$ have indicated that PrEP is a promising way to prevent HIV infection; however, poor MTC might limit its effectiveness. Thus, it would be important to identify ways of enhancing MTC to help maximise the benefits of PrEP in the real-world setting. Furthermore, our findings were based on trial level MTC obtained through assayed levels of study medication in blood samples, which is a more objective approach than other forms of MTC estimation, that is, self-reported MTC from questionnaires or counting the number and percentage of pills taken by participants. ${ }^{17}$

In the subgroup analyses, we found that the pooled MTC was lower in participants aged $<30$ years compared with the corresponding value in older participants. Similar results were also reported in Haberer et al's study of HIV-serodiscordant couples $^{18}$ and Grant et al's study of MSM. ${ }^{15}$ Young subjects in specific high-risk populations, such as MSM, are known to have a higher HIV infection risk than their older counterparts. ${ }^{19}$ Our results emphasise that more attention should be paid to the young subpopulation at risk of HIV infection; in these individuals, MTC education is especially important for the success of PrEP in order to maximise the drug efficacy.

Good MTC of PrEP requires not only adequate management of the participants themselves, but should also take into account social and environmental factors, especially for women. ${ }^{72021}$ Our finding that women participants had a lower level of MTC than that of IDU, sexually active heterosexual adults, MSM and transgender individuals, as well as serodiscordant couples, echoes the

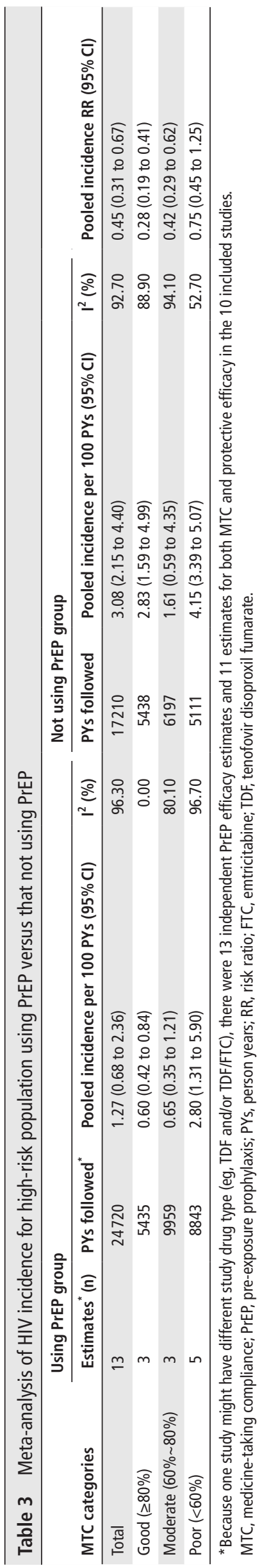


outcomes of Celum et al's study. ${ }^{22}$ The preexposure prophylaxis trial for HIV prevention among African women (FEM-PrEP) trial reported that there are some culturally specific barriers, such as stigma about sexual activity which may explain the low PrEP MTC in women, ${ }^{23}$ and furthermore, in some countries, women may have only limited access to reproductive health services. ${ }^{22}$ These findings highlight that MTC may be improved by initiating support programmes for women to eliminate these barriers and that these should be integrated into PrEP programmes to increase their likelihood of oral PrEP drug usage. Furthermore, before embarking on formal PrEP, it is necessary to provide female subjects with more education and guidance about the importance of MTC for the success of PrEP.

In addition, our results from the medication intensity subgroup analysis indicated that the intermittent regimen group had better MTC than daily medication group (85.8\% vs $56.1 \%)$, although there was only a marginal significant difference between the two groups, which might be caused by the small sample size with low statistical power (only one intermittent dosage study included in our analysis). The daily oral PrEP strategy as recommended by the CDC in the USA ${ }^{2}$ has proved to be a major challenge in reallife clinical practice because of the MTC and of the potential adverse effects of the therapy on liver and kidney functions, ${ }^{24} 25$ as well as in reducing bone mineral density. ${ }^{26}$ Recently, an intermittent PrEP medication strategy (ie, taking the medication before and after sexual intercourse) has been advocated as one way to improve the protective efficacy by elevating the MTC since the individual has to consume less medication while still enjoying a good $(86 \%)$ protective effect. ${ }^{12}$ These findings indicate that intermittent PrEP regimen strategy should be evaluated in future trials to confirm its efficiency in comparison to the daily regimen. For example, in an HIV high-risk population who were willing to initiate PrEP but have low likelihood of achieving a daily PrEP strategy, the more convenient intermittent regimen strategy might be recommended in clinical practice.

By integrating the published RCT studies, we also found that there was a significant positive association between MTC and protective efficacy for oral PrEP. A similar finding has also been reported in other PrEP studies. ${ }^{15} 23$ 27-30 For example, Grant et $a l^{15}$ stated that HIV incidence was reduced by $73 \%$ with high MTC ( $\geq 90 \%$ of doses), but only by $50 \%$ with intermediate MTC ( $\geq 50 \%$ of doses) and by even less, $32 \%$ with low MTC $(<50 \%$ of doses), which is very close to our results. The clarified relationship between MTC and PrEP efficacy should be taken into account in the development of PrEP medication strategies

\section{Key messages}

- The integrated medicine-taking compliance (MTC) in individuals at high risk of HIV infection was almost moderate $(59.9 \%(95 \% \mathrm{Cl} 43.1 \%$ to $74.6 \%))$.

- The MTC of participants aged $<30$ years was lower than those equal or older than 30 years $(34.9 \%$ vs $69.6 \%$, $\mathrm{p}<0.001)$.

- Studies that enrolled only women as participants had lower MTC than those recruiting either only men or both men and women $(31.3 \%$ vs $71.7 \%$ and $31.3 \%$ vs $71.0 \%$, all $p<0.01)$.

- The HIV infection risk increased as the MTC declines, with the incidence risk ratios being $0.28(95 \% \mathrm{Cl} 0.19$ to 0.41$), 0.42$ $(95 \% \mathrm{Cl} 0.29$ to 0.62$)$ and $0.75(95 \% \mathrm{Cl} 0.45$ to 1.25$)$ in the good $(\geq 80 \%)$, moderate $(60 \% \sim 80 \%)$ and poor $(<60 \%)$ MTC subgroups (linear trend test $p<0.01$ ). as it would help to optimise the medical resource allocation so as to promote the benefits of oral PrEP.

Although we have estimated the pooled MTC proportion of oral PrEP, which is very important for PrEP usage in the real world, there were two limitations in our study. First, our pooled results only evaluated oral PrEP studies, but did not include other PrEP dosing routes, such as intramuscular injection, intravenous infusion and so on. Second, most of the included studies were performed in high HIV infection risk population living in Africa, with only one from Asia which could not represent the PrEP MTC in other continents.

In summary, our findings indicate that the MTC of oral PrEP for HIV prevention was almost moderate, although younger and female subjects had a lower level of MTC. There is a positive correlation between MTC level and PrEP protective efficacy. These participants in PrEP trials should receive encouragement, training and guidance of the importance of MTC before project initiating, which is particularly crucial in female and in younger subjects.

\section{Handling editor Jackie A Cassell}

Acknowledgements We appreciate all the researchers whose articles were included in the study.

Contributors The work presented here was carried out in collaboration between all authors. KY and JJX contributed equally to the work. KY and JML worked on data collection and data abstraction. KY analysed the data, interpreted the results and wrote the paper. Others supplied comments and helped with interpretation of the results.

Funding This study was supported by the Mega-projects of national science research for the 12th Five-Year Plan (2012ZX10001-006).

Competing interests None declared.

Provenance and peer review Not commissioned; externally peer reviewed.

(C) Article author(s) (or their employer(s) unless otherwise stated in the text of the article) 2018. All rights reserved. No commercial use is permitted unless otherwise expressly granted.

\section{REFERENCES}

1 UNAIDS. The gap report. 2014 http://files.unaids.org/en/media/unaids/contentassets/ docum-ents/unaidspublication/2014/UNAIDS_Gap_report_en.pdf (accessed 15 Dec 2016).

2 CDC. Preexposure prophylaxis for the prevention of HIV infection in the United States-2014: a clinical practice guideline, 2014. https://www.cdc.gov/hiv/pdf/ prepguidelines2014.pdf (accessed 15 Dec 2016).

3 Marrazzo JM, Ramjee G, Richardson BA, et al. Tenofovir-based preexposure prophylaxis for HIV infection among african women. N Engl J Med 2015;372:509-18

4 Van Damme L, Corneli A, Ahmed K, et al. Preexposure prophylaxis for HIV infection among african women. N Eng/ J Med 2012;367:411-22.

5 Donnell D, Baeten JM, Bumpus NN, et al. HIV protective efficacy and correlates of tenofovir blood concentrations in a clinical trial of PrEP for HIV prevention. J Acquir Immune Defic Syndr 2014;66:340-8.

6 Van de Mortel TF. Faking it: social desirability response Bias in self-report research. Aust J Adv Nurs 2008;25:40-8

7 Grant RM, Anderson PL, McMahan V, et al. Uptake of pre-exposure prophylaxis, sexual practices, and HIV incidence in men and transgender women who have sex with men: a cohort study. Lancet Infect Dis 2014;14:820-9.

8 Moher D, Liberati A, Tetzlaff J, et al. Preferred reporting items for systematic reviews and meta-analyses: the PRISMA statement. Ann Intern Med 2009;151:264-9.

9 Jadad AR, Moore RA, Carroll D, et al. Assessing the quality of reports of randomized clinical trials: is blinding necessary? Control Clin Trials 1996;17:1-12.

10 Lipsey MW, Wilson DB. Practical meta-analysis, 2001.

11 Higgins JP, Thompson SG. Quantifying heterogeneity in a meta-analysis. Stat Med 2002:21:1539-58.

12 Molina JM, Capitant C, Spire B, et al. On-Demand Preexposure Prophylaxis in Men at High Risk for HIV-1 infection. N Engl J Med 2015;373:2237-46.

13 García-Lerma JG, Otten RA, Qari SH, et al. Prevention of Rectal SHIV transmission in macaques by Daily or Intermittent prophylaxis with emtricitabine and tenofovir. PLOS Med 2008:5:e28

14 Choopanya K, Martin M, Suntharasamai P, et al. Antiretroviral prophylaxis for HIV infection in injecting drug users in Bangkok, Thailand (the Bangkok Tenofovir 
Study): a randomised, double-blind, placebo-controlled phase 3 trial. Lancet 2013;381:2083-90.

15 Grant RM, Lama JR, Anderson PL, et al. Preexposure chemoprophylaxis for HIV prevention in men who have sex with men. N Engl J Med 2010;363:2587-99.

16 Baeten JM, Donnell D, Ndase P, et al. Antiretroviral prophylaxis for HIV Prevention in Heterosexual Men and Women. N Engl J Med Overseas Ed 2012;367:399-410.

17 Musinguzi N, Muganzi CD, Boum Y, et al. Comparison of subjective and objective adherence measures for preexposure prophylaxis against HIV infection among serodiscordant couples in East Africa. AIDS 2016;30:1121-9.

18 Haberer JE, Baeten JM, Campbell J, et al. Adherence to antiretroviral prophylaxis for HIV prevention: a substudy cohort within a clinical trial of serodiscordant couples in East Africa. PLoS Med 2013;10:e1001511.

19 Balaji AB, Bowles KE, Le BC, et al. High HIV incidence and prevalence and associated factors among young MSM, 2008. AIDS 2013;27:269-78.

20 Amico KR. Adherence to preexposure chemoprophylaxis: the behavioral bridge from efficacy to effectiveness. Curr Opin HIV AIDS 2012;7:542-8.

21 Kippax S, Stephenson N. Beyond the distinction between biomedical and social dimensions of HIV prevention through the Lens of a social public health. Am J Public Health 2012;102:789-99.

22 Celum CL, Delany-Moretlwe S, McConnell M, et al. Rethinking HIV prevention to prepare for oral PrEP implementation for young african women. J Int AIDS SoC 2015; 18:20227.

23 Amico KR, Mansoor LE, Corneli A, et al. Adherence support approaches in biomedical HIV prevention trials: experiences, insights and future directions from four multisite prevention trials. AIDS Behav 2013;17:2143-55.

24 Solomon MM, Lama JR, Glidden DV, et al. Changes in renal function associated with oral emtricitabine/tenofovir disoproxil fumarate use for HIV pre-exposure prophylaxis. AIDS 2014:28:851-9.
25 Van Damme L, Corneli A, Ahmed K, et al. Preexposure prophylaxis for HIV infection among african women. N Engl J Med 2012;367:411-22.

26 Mulligan K, Glidden DV, Anderson PL, et al. Effects of Emtricitabine/Tenofovir on Bone Mineral Density in HIV-Negative persons in a Randomized, Double-Blind, PlaceboControlled Trial. Clin Infect Dis 2015;61:572-80.

27 Kashuba AD, Patterson KB, Dumond JB, et al. Pre-exposure prophylaxis for HIV prevention: how to predict success. Lancet 2012;379:2409-11.

28 Dimitrov DT, Mâsse BR, Donnell D. PrEP adherence patterns strongly affect individual HIV risk and Observed Efficacy in Randomized clinical trials. J Acquir Immune Defic Syndr 2016;72:444-51.

29 Baeten JM, Donnell D, Ndase P, et al. Antiretroviral prophylaxis for HIV prevention in heterosexual men and women. N Eng/ J Med 2012;367:399-410.

30 van der Straten A, Van Damme L, Haberer JE, et al. Unraveling the divergent results of pre-exposure prophylaxis trials for HIV prevention. AIDS 2012;26:F13-19.

31 Baeten JM, Donnell D, Mugo NR, et al. Single-agent tenofovir versus combination emtricitabine plus tenofovir for pre-exposure prophylaxis for HIV-1 acquisition: an update of data from a randomised, double-blind, phase 3 trial. Lancet Infect Dis 2014;14:1055-64.

32 McCormack S, Dunn DT, Desai M, et al. Pre-exposure prophylaxis to prevent the acquisition of HIV-1 infection (PROUD): effectiveness results from the pilot phase of a pragmatic open-label randomised trial. Lancet 2016;387:53-60.

33 Thigpen MC, Kebaabetswe PM, Paxton LA, et al. Antiretroviral preexposure prophylaxis for heterosexual HIV transmission in Botswana. N Eng/ J Med 2012;367:423-34

34 Peterson L, Taylor D, Roddy R, et al. Tenofovir disoproxil fumarate for prevention of HIV infection in women: a phase 2, double-blind, randomized, placebo-controlled trial. PLoS Clin Trials 2007;2:e27. 